OPEN ACCESS

Edited by:

Marcel van der Heyden,

University Medical Center Utrecht,

Netherlands

Reviewed by:

David R. Van Wagoner,

Cleveland Clinic Lerner College

of Medicine, United States

Tong Liu,

Tianjin Medical University, China

*Correspondence:

Sunny S. PO

sunny-po@ouhsc.edu

Hong Jiang

whujianghong@163.com

†These authors have contributed equally to this work

Specialty section:

This article was submitted to Cardiac Electrophysiology, a section of the journal

Frontiers in Physiology

Received: 04 April 2018

Accepted: 20 July 2018

Published: 21 August 2018

Citation:

Zhou X, Wang Z, Huang B, Yuan S,

Sheng X, Yu L, Meng G, Wang Y, Po SS and Jiang $H$ (2018) Regulation of the NRG1/ErbB4 Pathway in the Intrinsic Cardiac Nervous System Is a Potential Treatment for Atrial Fibrillation

Front. Physiol. 9:1082 doi: 10.3389/fphys.2018.01082

\section{Regulation of the NRG1/ErbB4 Pathway in the Intrinsic Cardiac Nervous System Is a Potential Treatment for Atrial Fibrillation}

\author{
Xiaoya Zhou't, Zhuo Wang't, Bing Huang ${ }^{1}$, Shenxu Yuan', Xia Sheng' ${ }^{2}$, Lilei Yu', \\ Guannan Meng ${ }^{1}$, Yuhong Wang ${ }^{1}$, Sunny S. Po ${ }^{3 *}$ and Hong Jiang ${ }^{1 *}$
}

${ }^{1}$ Department of Cardiology, Renmin Hospital of Wuhan University, Wuhan, China, ${ }^{2}$ Sir Run Run Shaw Institution of Clinical Medicine and Department of Cardiology, Sir Run Run Shaw Hospital Affiliated to Medical College of Zhejiang University, Hangzhou, China, ${ }^{3}$ Heart Rhythm Institute, University of Oklahoma Health Sciences Center, Oklahoma City, OK, United States

Background: The NRG1/ErbB4 signaling mechanism has been widely studied in the central nervous system for many years. However, the role of this pathway in modulating the intrinsic cardiac nervous system is largely unknown.

Objective: The present study investigated whether the NRG1/ErbB4 signaling system affects the activity of major atrial ganglionated plexi (GP) in a paroxysmal atrial fibrillation (AF) model by 6-h rapid atrial pacing (RAP).

Methods: Twenty-four dogs were randomly divided into (1) a control group (saline microinjections into GP), (2) RAP group (saline microinjections into GP plus 6 h-RAP), (3) NRG1 group (microinjections of neuregulin-1 into GP plus 6 h-RAP) and (4) NRG1 + ERA group (microinjections of neuregulin-1 and ErbB4 receptor antagonist-ERA into GP plus 6 h-RAP). The effective refractory period (ERP), window of vulnerability (WOV), anterior right GP (ARGP) function and neural activity were measured. ARGP tissues were excised for histological study and western blotting.

Results: When compared to the control group, 6 h-RAP produced a significant (1) decrease in ERP, an increase in $\Sigma$ WOV, (2) an increase in ARGP neural activity and neural function, and (3) an increase in c-fos and nerve growth factor protein expression in the ARGP. However, microinjection of NRG1 into the ARGP prior to RAP prevented ERP shortening and AGRP activity enhancement and inhibited the expression of c-Fos and NGF proteins. Furthermore, these changes were significantly attenuated by pretreatment with an ErbB4 receptor antagonist.

Conclusion: The NRG1/ErbB4 signaling pathway may exist in the GP, and activation of this pathway suppressed RAP-induced GP activation, atrial electrical remodeling and AF.

Keywords: neuregulin-1, ErbB4, atrial fibrillation, autonomic nervous system, ganglionated plexi 


\section{INTRODUCTION}

The extrinsic and intrinsic autonomic nervous systems control the heart. The intrinsic autonomic nervous system consists of multiple ganglionated plexi (GP), which are called the heart's 'little brain' (Wake and Brack, 2016). Numerous preclinical studies have revealed that hyperactivity of the major atrial GP plays a crucial role in the initiation and maintenance of atrial fibrillation (AF) (Benjamin et al., 1998; Lu et al., 2008; Tan et al., 2008; Park et al., 2012). Interventions that inhibit GP neural activity or directly injure the GP neurons suppress AF in animal models and patients ( $\mathrm{Lu}$ et al., 2008; Katritsis et al., 2013; Yu et al., 2013; Stavrakis et al., 2015; Lo et al., 2016). However, the mechanism(s) underlying GP inhibition are not clear.

Neuregulins (NRGs) play an important role in neural development and brain activity homeostasis. NRG-1 is distributed widely in the central and peripheral nervous systems (Buonanno and Fischbach, 2001; Thompson et al., 2007). ErbB4 is the only known receptor that exhibits high affinity for NRG1 and an active tyrosine kinase domain (Tan et al., 2011). NRG1/ErbB4 signaling was implicated in neural development, including circuitry generation, axon ensheathment, neurotransmission, and synaptic plasticity (Buonanno and Fischbach, 2001). The signaling pathway has also been shown to regulate neuronal excitability and synaptic plasticity in the adult brain (Corfas et al., 2004; Mei and Nave, 2014).

The NRG1/ErbB4 signaling networks function widely in the central nervous system, and NRG1 suppresses excitatory neuronal activity via activation of the ErbB4 receptor (Fazzari et al., 2010; Tan et al., 2011). A previous study found a significant association between NRG-1 levels and the presence of paroxysmal AF (Shao et al., 2014). We hypothesized that an NRG1/ErbB4 signaling pathway exists in the cardiac nervous system (GP), and activation of this signaling pathway influences $\mathrm{AF}$ initiation and maintenance.

\section{MATERIALS AND METHODS}

All experimental protocols conformed to the Guideline for the Care and Use of Laboratory Animals published by the United States National Institutes of Health (NIH Publication, revised 1996), and the Committee on the Ethics of Animal Experiments of Wuhan University approved all protocols.

\section{Animal Preparation}

Twenty-four canines weighing 20-25 kg were included in this study. All surgeries were performed under anesthesia with $3 \%$ sodium pentobarbital with an initial dose of $1 \mathrm{ml} / \mathrm{kg}$ (IV) and a maintenance dose of $2 \mathrm{ml} / \mathrm{h}$ (IV). Body surface electrocardiography and blood pressure were recorded using a computer-based laboratory system (Lead7000, Jinjiang Inc., Chengdu, China). The core body temperature of the dogs was maintained at $36.5^{\circ} \mathrm{C} \pm 1.5^{\circ} \mathrm{C}$. Bilateral thoracotomy was performed at the fourth intercostal space to expose the bilateral atria, atrial appendages, pulmonary veins and atrial GP.
Multielectrode catheters were sutured at the following seven sites: right superior pulmonary vein (RSPV), right inferior pulmonary vein (RIPV), right atrium (RA), right atrial appendage (RAA), left superior pulmonary vein (LSPV), left inferior pulmonary vein (LIPV), and left atrium (LA). High frequency stimulation $(20 \mathrm{~Hz}$, 0.1 -ms pulse duration) was applied to identify the location of the major GPs.

\section{Programmed Stimulation}

Six hours of RAP ( $20 \mathrm{~Hz}, 2 \times$ threshold) was delivered at the left atrial appendage to induce acute atrial remodeling (Sheng et al., 2011). RAP was temporarily stopped for 5-10 $\mathrm{min}$ to measure the effective refractory period (ERP). ERP was determined using a programmed stimulation that consisted of eight consecutive stimuli $(\mathrm{S} 1-\mathrm{S} 1$ interval $=330 \mathrm{~ms})$ followed by a premature stimulus (S1-S2 interval). The S1-S2 intervals were decreased from $150 \mathrm{~ms}$ initially in decrements of $10 \mathrm{~ms}$, then $2 \mathrm{~ms}$ when approaching ERP. We used the window of vulnerability (WOV) as a quantitative measure of AF inducibility. The interval between the longest and shortest S1-S2 interval (in ms) at which AF was induced was defined as the WOV. The cumulative WOV $(\Sigma$ WOV) was the sum of the WOVs at all sites in each dog.

\section{Measurement of Anterior Right GP (ARGP) Function}

Four major GPs were located anatomically. The anterior right GP (ARGP) was located at the right superior PV - atrial junction; the inferior right GP (IRGP) was situated at the junction of inferior vein cava and both atria; the superior left GP (SLGP) was located near the left superior PV - atrial junction and left pulmonary artery; and the inferior left GP (ILGP) was located at the left inferior PV - atrial junction. The ARGP was precisely localized via application of high-frequency stimulation $(20 \mathrm{~Hz}$, square wave pulse, $0.1 \mathrm{~ms}$ duration, 2-5 V) using a Grass stimulator (model S88, Grass Instruments, Quincy, MA, United States). ARGP function was measured as the maximal change in sinus rate (SR) in response to direct electrical stimulation of ARGP (Sha et al., 2011; Wang et al., 2016). We used four incremental voltage levels to stimulate the ARGP (level 1: 1-4 volts; level 2: 5-7.5 volts; level 3: 8-10 volts; level 4: 10-15 volts). Each high-frequency stimulation lasted $\leq 30 \mathrm{~s}$, and we waited until the SR returned to baseline before delivering the next round of stimulation. Data are presented as the percent of maximal SR slowing (compared to the baseline state) induced by ARGP stimulation at different levels of stimulation voltages.

\section{Measurement of GP Neural Activity}

A coated tungsten microelectrode $(2 \mathrm{~cm}$ in length) with an exposed tip of $50 \mu \mathrm{m}$ was inserted into the fat pad containing the ARGP, and an impedance of 9-12 M $\Omega$ at $1,000 \mathrm{~Hz}$ was mounted on a micromanipulator. Electrical signals generated by the ARGP were recorded using a PowerLab data acquisition system (8/35, ADInstruments, Bella Vista, New South Wales, Australia), and the signals were amplified using an amplifier (DP-304, Warner Instruments, Hamden, CT, United States) with bandpass filters set at $300 \mathrm{~Hz}$ to $1 \mathrm{kHz}$ and an amplification range of $30-50$ 


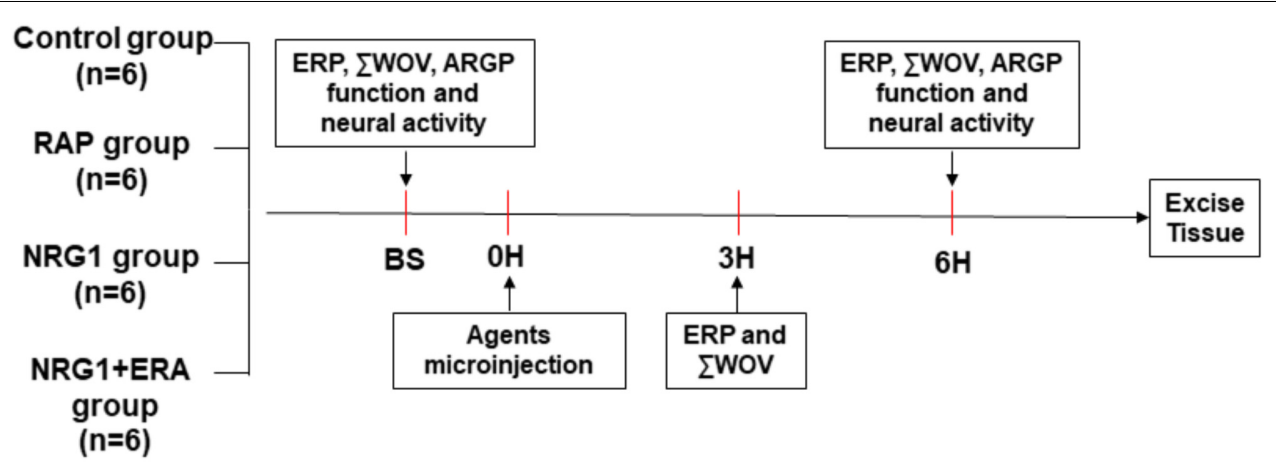

FIGURE 1 | Flow diagram of the present study. RAP was performed after saline, NRG1 or NRG1 plus ERA microinjection into four atrial GP and LOM, except in the control group. ERP and $\Sigma$ WOV at atrial and pulmonary vein sites were examined at baseline, the end of 3rd hour and the end of the 6th hour. ARGP function and neural activity were examined at baseline and the end of 6th hour, respectively. GP tissues were excised for histological studies at the end of the 6th hour.

times (Yu et al., 2013). Neural activity was characterized using the recorded amplitude and frequency that was used to quantitate neural activity. Neural activity was defined as deflections with a signal-to-noise ratio greater than 3:1 (Yu et al., 2014).

\section{Experimental Protocol}

Twenty-four dogs were randomly divided into the control group, RAP group, NRG1 group, and NRG1 plus the ErbB4 receptor antagonist ERA (NRG1 + ERA) group (Figure 1). Saline was microinjected into the four major GP (ARGP, inferior right GP, superior left GP and inferior left GP) and the ligament of Marshall (LOM) followed by $6 \mathrm{~h}$ of sham RAP in the control group. Saline was microinjected into the four major GPs and the LOM followed by $6 \mathrm{~h}$ of RAP in the RAP group. NRG1 $(10 \mu \mathrm{M})$ was microinjected into the four major GP and the LOM followed by $6 \mathrm{~h}$ of RAP in the NRG1 group. NRG1 $(10 \mu \mathrm{M})$ and ERA (PD158780, an ErbB4 receptor antagonist, $2 \mathrm{mM}$ ) were co-injected into the four major GP and the LOM followed by $6 \mathrm{~h}$ of RAP in the NRG1 + ERA group. All agents were administered into the GP and LOM location using a $34 \mathrm{G}$ stainless steel needle connected to a micropump (Longer Precision Pump TJ-4A, Hebei, China) at a rate of $0.5 \mu \mathrm{l} / \mathrm{min}$ in a total volume of 5-10 $\mu \mathrm{l}$. Atrial ERP and WOV were determined at baseline and the end of the 3rd and 6th hours of RAP. ARGP function and ARGP neural activity were measured at baseline and the end of the 6th hour of RAP (Figure 1).

\section{Western Blot Analysis and Histological Studies}

Anterior right GP was excised and washed with saline at the end of the experiment. Tissue for western blot analysis was dissected into small portions and maintained at $-80^{\circ} \mathrm{C}$ for subsequent analyses. Tissue for histological studies was fixed with $4 \%$ paraformaldehyde. The protein expression levels of c-fos and nerve growth factor (NGF) in ARGP were analyzed using western blot analysis. The primary antibodies used were anti-c-fos (Abcam, Cambridge, England) and anti-NGF (Santa Cruz, Dallas, TX, United States). The membrane was washed and incubated with secondary anti-rabbit antibodies at $37^{\circ} \mathrm{C}$ for $2 \mathrm{~h}$. Antibody-binding protein bands were visualized and quantified. Immunofluorescence staining was used to confirm the expression and location of ErbB4 in atrial GP. Paraffin-embedded GP tissues were cut transversely into $5-\mu \mathrm{m}$ sections. The primary antibodies used were anti-ErbB4 (Santa Cruz, Dallas, TX, United States) and anti-parvalbumin (Abcam, Cambridge, England). Quantitative analyses were performed using commercially available software (Image Pro Plus, Media Cybernetics, Inc., Rockville, MD, United States).

\section{Statistical Analysis}

Continuous variables are presented as the means \pm standard deviations (SD). A one-way analysis of variance (ANOVA) followed by Tukey's test was used for post hoc multiple comparisons of c-fos, NGF, ErbB4, and parvalbumin levels between different groups. A two-way ANOVA followed by Tukey's test was used to examine differences in ERP, $\Sigma$ WOV, ARGP function and ARGP neural activity. SPS22.0 (SPSS Inc., Chicago, IL, United States) was used for data analysis and graphing, and two-tailed $P<0.05$ was considered significant.

\section{RESULTS}

\section{ERP and $\Sigma$ WOV}

No significant difference in ERP was demonstrated at any site among all groups. The $6 \mathrm{~h}$-RAP treatment resulted in a significant decrease in the ERP during the first $3 \mathrm{~h}$ of RAP at all pulmonary vein and atrial recording sites (Figure 2). Microinjection of NRG1 prevented ERP shortening and $\Sigma$ WOV increase. However, the effects of NRG1 were blocked in the NRG1 + ERA group. The ERP at all sites was significantly shortened, and the $\Sigma$ WOV was significantly increased (Figure 2).

\section{GP Function}

A significant slowing of SR was observed at baseline because of the high-frequency stimulation of the ARGP in all groups 


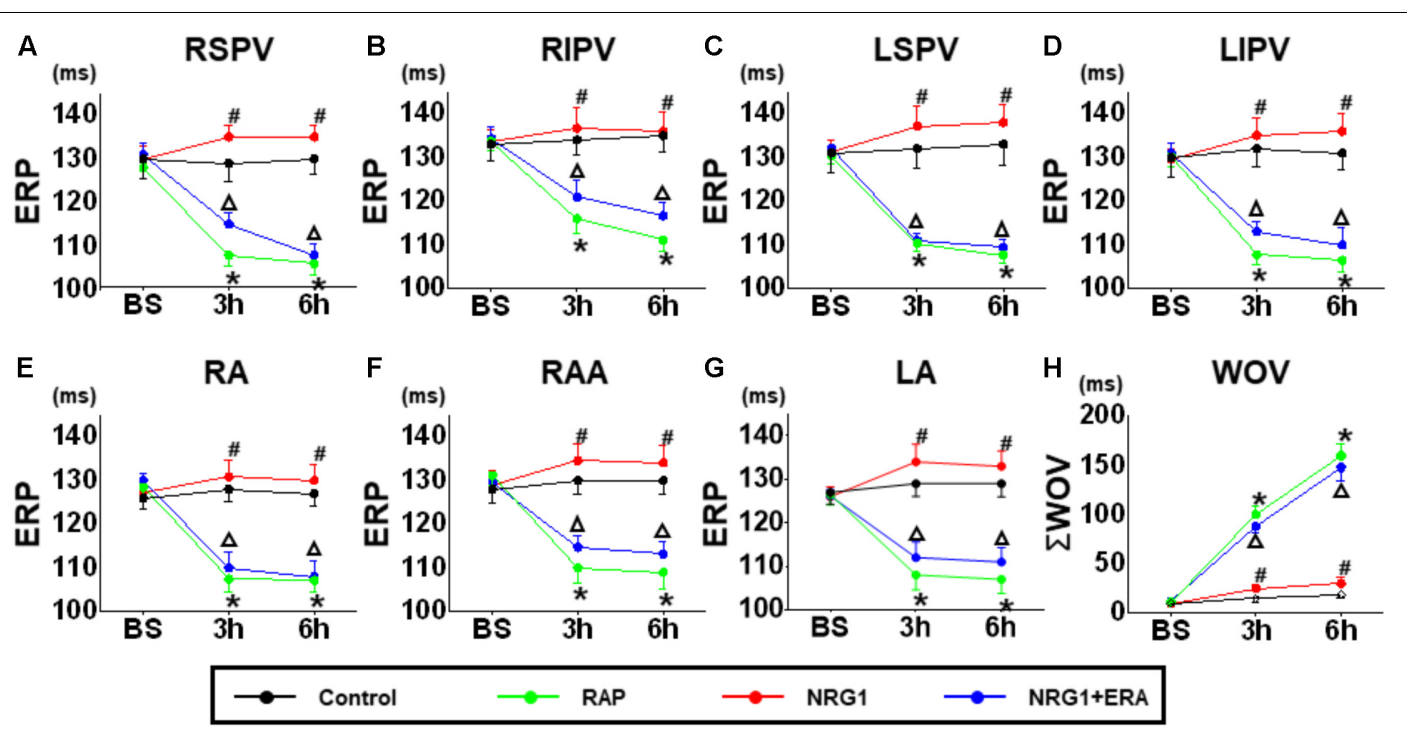

FIGURE 2 | Changes in ERP and $\Sigma$ WOV. (A-G) The ERP changes at different time points in the control group, RAP group, NRG1 group and NRG1 + ERA group. (H) The $\Sigma$ WOV changes at different time points in the four groups. The ERP was shortened, and $\Sigma$ WOV was widened in atrial and pulmonary vein sites in the RAP group. NRG1 alleviated the effect of RAP, and ERA blocked the beneficial effects of NRG1 on ERP and $\Sigma$ WOV. * $p<0.05$ vs. NRG1 group. ${ }^{*} p<0.05$ vs. RAP group. ${ }^{\Delta} P<0.05$ vs. the NRG1 group.

A

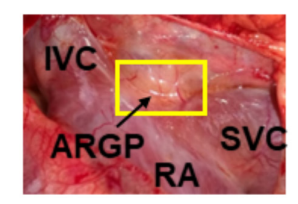

C

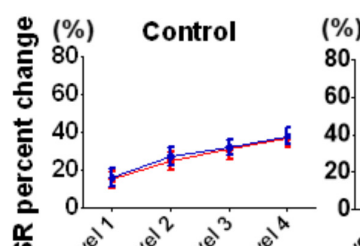

D

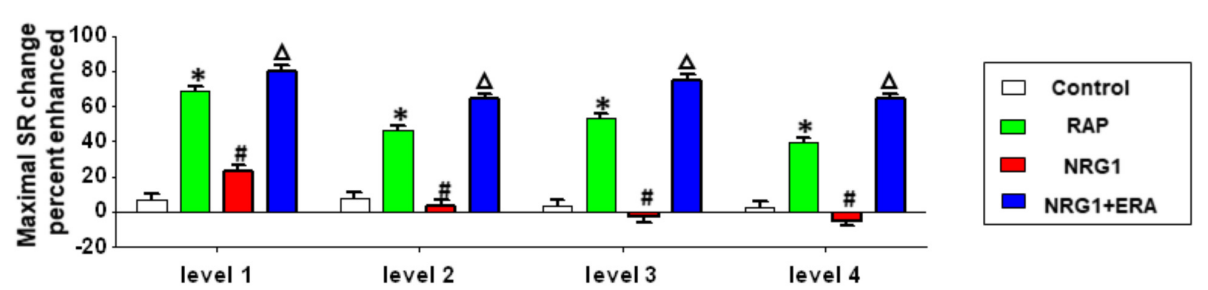

B

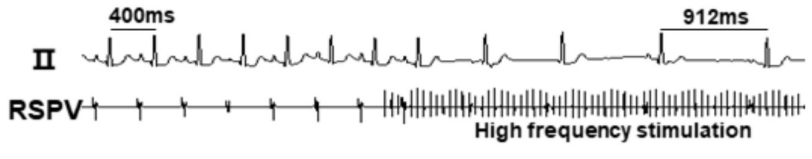
High frequency stimulation

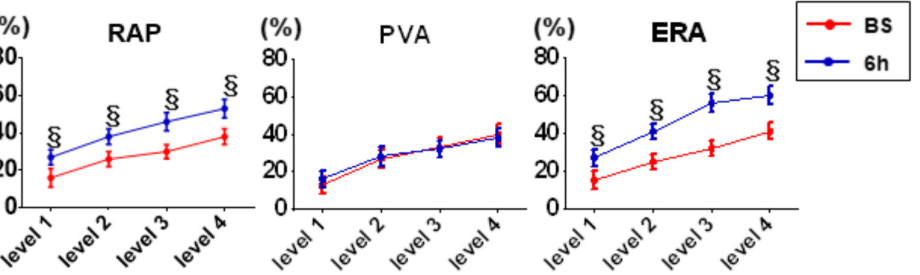

FIGURE 3 | Changes in ARGP function. (A) The position of ARGP. (B) Representative example of the SR slowing response to ARGP stimulation. (C) ARGP function assessed using the SR slowing responses to ARGP stimulation before and after $6 \mathrm{~h}$ of RAP. (D) Percent of maximal SR change induced by different interventions after 6 h of RAP. ${ }^{\S} P<0.05$ vs. baseline; ${ }^{*} P<0.05$ vs. the control group; ${ }^{\#} P<0.05$ vs. the RAP group; ${ }^{\Delta} P<0.05$ vs. the NRG1 group.

(Figure 3). The ability of ARGP stimulation to slow SR was significantly augmented after $6 \mathrm{~h}$ of RAP in the RAP and NRG1 + ERA groups (Figure 3C). Figure 3D illustrates that the SR slowing responses were augmented by $6 \mathrm{~h}$ of RAP at all levels of ARGP stimulation, but NRG-1 microinjection prevented these augmented responses. However, ERA and NRG1 co-injection produced similar effects as RAP alone.

\section{GP Neural Activity}

Figure 4A shows the changes of ARGP neural activity in each group. There was no significant difference at baseline among all groups. No significant difference was found at different time points in the control group. In the RAP group, $6 \mathrm{~h}$-RAP resulted in a significant increase in ARGP neural activity compared to baseline. However, Figures $4 \mathrm{~A}-\mathrm{C}$ shows that RAP-induced 

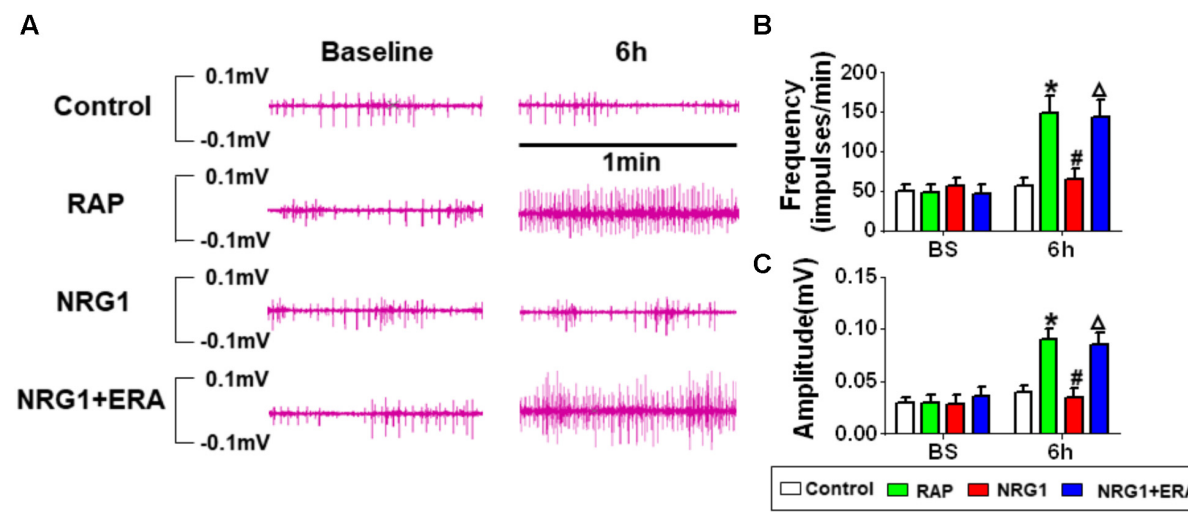

FIGURE 4 | Changes in ARGP neural activity. (A) Representative ARGP neural activity in the control, RAP group, NRG1 group and NRG1 + ERA group. (B) Quantitative analysis of the frequency of ARGP neural spikes. (C) Quantitative analysis of the amplitude of ARGP neural spikes. * $P<0.05$ vs. the control group; ${ }^{\#} P<0.05$ vs. the RAP group; ${ }^{\Delta} P<0.05$ vs. the NRG1 group.

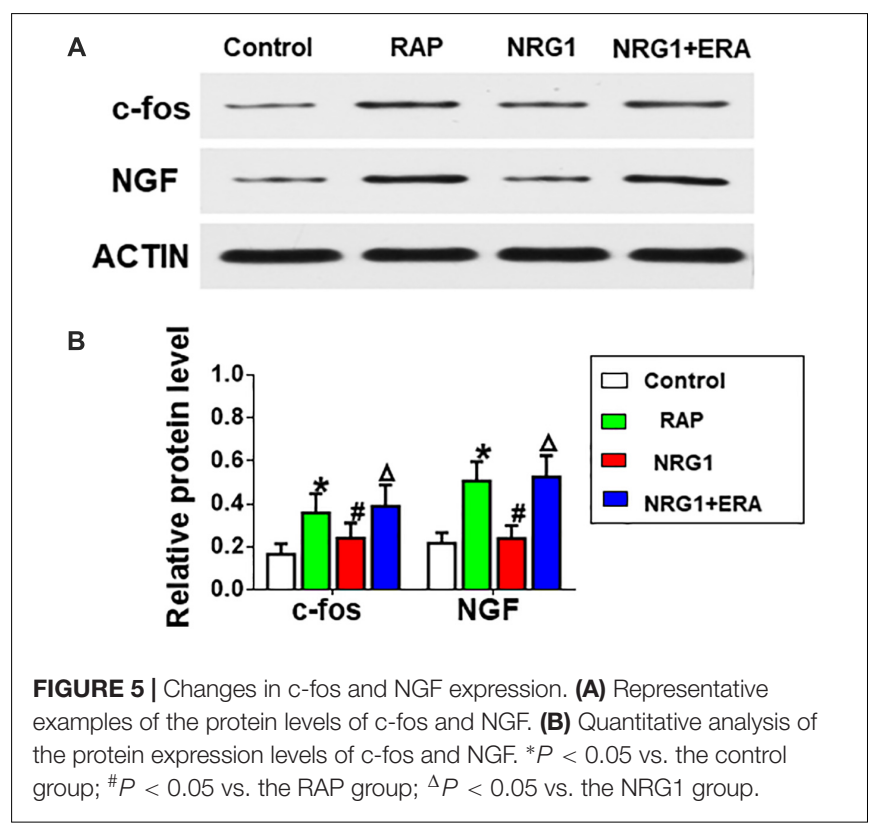

ARGP neural activation was prevented in the NRG1 group (both in frequency and amplitude). However, ARGP activity increased significantly in the NRG1 + ERA group compared to that in the NRG1 group.

\section{C-fos and NGF}

The protein levels of $\mathrm{c}$-fos and NGF in the ARGP were much higher in the RAP group than in the control group. However, NRG1 microinjection significantly attenuated the RAP-induced expression of c-fos and NGF protein, and the ErbB4 receptor antagonist ERA prevented the inhibitory effects (Figure 5).

\section{Parvalbumin (PAV)-Positive Neurons and ErbB4 Receptor}

Immunofluorescence staining of PAV and DAPI indicated the presence of PAV-positive neurons in atrial GP, and the expression of PAV-positive neurons decreased markedly after 6 h-RAP (Figure 6). In contrast, 6 h-RAP did not affect the high levels of ErbB4 expression.

\section{DISCUSSION}

\section{Major Finding}

NRG1/ErbB4 signaling has been widely studied in the central nervous system for many years (Hu et al., 2014; GonzalezBurgos et al., 2015). To the best of our knowledge, this study is the first report of the NRG1/ErbB4 signaling pathway in the cardiac autonomic nervous system. Local microinjected NRG1 may inhibit the activity and function of ARGP and decrease AF inducibility. An ErbB4 receptor antagonist (PD158780) abolished the protective effect of NRG1, which indicates that NRG1 functions via the ErbB4 receptor-related signaling pathway.

\section{The Possible Mechanism of the NRG1/ErbB4 Signaling Pathway in AF}

Most intrinsic nerve cell bodies reside in the fat pads on the epicardial surface, with axons that form connections with other nearby neurons, which create networks that appear as GP. The GP are connected with stellate ganglia in the thoracic and lumbar spine dorsal root ganglia (Kummer and Oberst, 1993; Tsai et al., 2017), which connect with the posterior and lateral hypothalamic nuclei and locus coeruleus in the brain stem (Jansen et al., 1995). The widespread distribution of these ganglia and the extensive networks of the plexuses suggest that intrinsic nerves play an important role in the initiation and maintenance of AF (Chen et al., 2014).

NRG1 is an expressed epidermal growth factor (EDF)-like protein, and it is essential for the normal development of the nervous system. NRG1 regulates target cell differentiation (Falls, 2003), neurotransmitter receptor expression (Buonanno and Fischbach, 2001), interneuronal peripheral nerve synapse development (Meyer and Birchmeier, 1995) and Schwann cell survival (Escher et al., 2005) in the peripheral nervous system. 


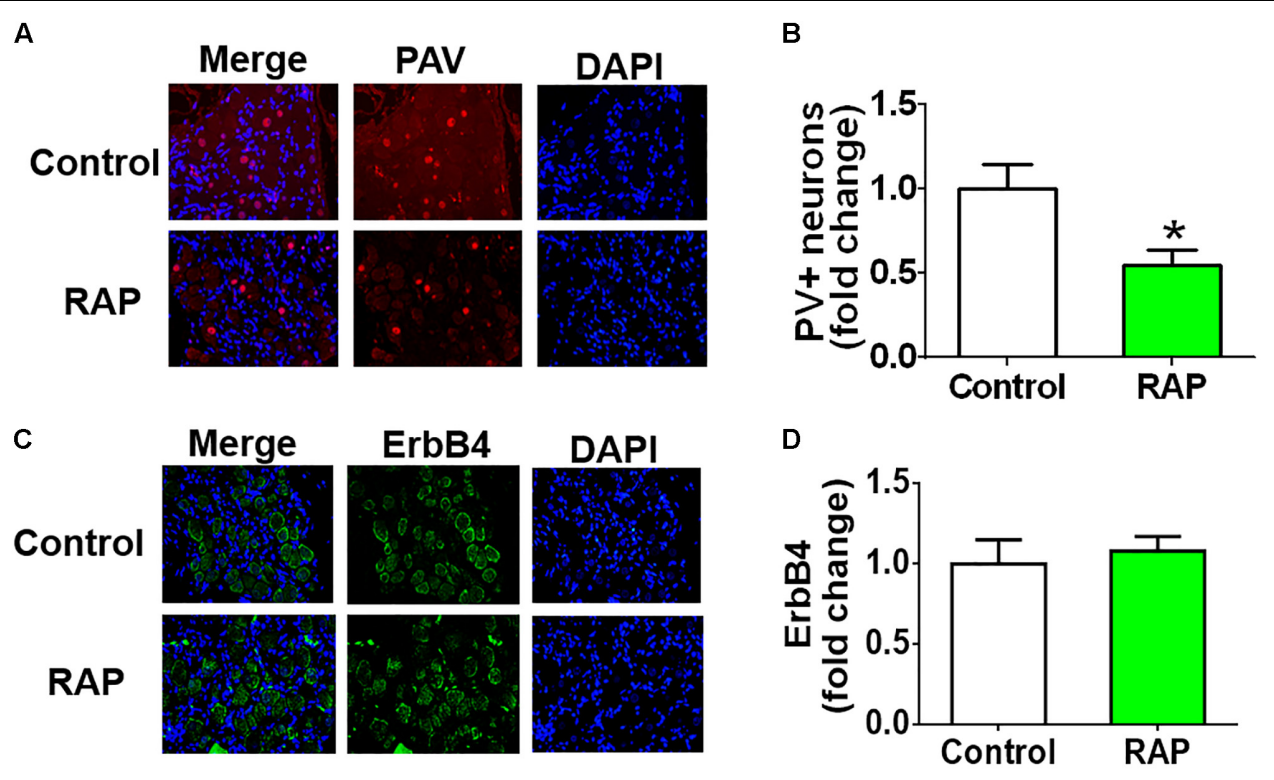

FIGURE 6 | Parvalbumin (PAV) and ErbB4 staining before and after $6 \mathrm{~h}$ of RAP. (A) Representative example of PAV-immunoreactive neurons (40×, red) within the ARGP. (B) Quantitative analysis of the PAV-positive neurons. (C) Representative example of ErbB4-immunoreactive neurons (40x, green) within the ARGP. (D) Quantitative analysis of the ErbB4 positive cells. * $p<0.05$ vs. control group.

The present study found that microinjection of extraneous NRG1 inhibited the activity and function of ARGP and decreased AF inducibility in a $6 \mathrm{~h}$-RAP-induced AF model in canines. We speculated that NRG-1 would exert protective effects in the maintenance of atrial electrophysiological stability. This hypothesis is consistent with a previous study indicating that exogenous NRG-1 administration exerted a neuroprotective effect in the central nervous system (Xu et al., 2005).

The activity of NRG1 is primarily attributed to the activation of ErbB receptors and induction of acetylcholine receptor expression (Falls, 2003; Mei and Xiong, 2008). Kuramochi et al. (2004) demonstrated that NRG-1 activated ErbB4 receptor phosphorylation in isolated cardiac myocytes. Our findings indicate that pretreatment with an ErbB4 receptor antagonist (PD158780) attenuated the protective effects of NRG1, which indicated that NRG1 inhibited GP neural hyperactivity via the ErbB4 receptor. We suggest that the ErbB4 receptor also exists in the cardiac autonomic nervous system, and NRG1 activation improves atrial electrophysiological stability via an ErbB4 receptor-related signaling pathway.

Previous studies have indicated that NRG1/ErbB4 expression was restricted to GABAergic interneurons, and its expression was particularly high in PAV-positive neurons (Vullhorst et al., 2009; Wen et al., 2010). Suzukawa et al. (1999) found that the loss of perikaryal PAV immunoreactivity and overexpression of c-fos and NGF were involved in the development of epileptogenesis after ethacrynic acid-induced seizure. C-fos is a rapid indicator of neuronal activation, and it is broadly used as a marker for fast neuronal activation (Dragunow and Robertson, 1987). NGF is a potential novel biomarker of sympathetic neurons, and it is a neurotrophic factor that initiates pathological sympathetic neural growth in damaged cardiac tissue (Lazarovici et al., 2006). Previous studies have indicated that NGF was related to increasing sympathetic tone, and autonomic intervention may suppress AF via inhibition of NGF expression (Zhou et al., 2004). We observed that 6h-RAP induced more expression of c-fos and NGF protein and PAV-positive neurons in the GP (Figure 6). The PAV-positive neurons may modulate the intrinsic cardiac autonomic nervous system after atrial electrical remodeling (Figure 7).

It is well known that eNOS activation and vagal modulation play a role in paroxysmal AF (Fatini et al., 2006; Yu et al., 2013). Activation of the M2 muscarinic cholinergic receptor activates the isoform of nitric oxide synthase in endothelial cells of cardiac muscle (Feron et al., 1997), and ErbB4 signaling is critical for eNOS activation and the modulation of vagally mediated changes in NO production (Feron et al., 1999). Our investigation demonstrated a potential mechanism of the NRG1/ErbB4 signaling system in a paroxysmal AF model. Sixhour RAP induced acute atrial electrical remodeling and AF via activation of the intrinsic cardiac autonomic nervous system ( $\mathrm{Lu}$ et al., 2008), and microinjection of NRG1 prevented activation of cardiac neural activity. The detailed mechanism by which ErbB4 and nitric oxide synthase function in paroxysmal AF is not known. More research is needed to further elucidate the detailed mechanism.

\section{Clinical Implications}

Atrial fibrillation is the most common arrhythmia, and its prevalence is expected to increase sharply as the population ages (Shen et al., 2011). A significant portion of these patients will exhibit drug-refractory AF and require ablation (Benjamin et al., 1998). Catheter or surgical ablation carries significant 

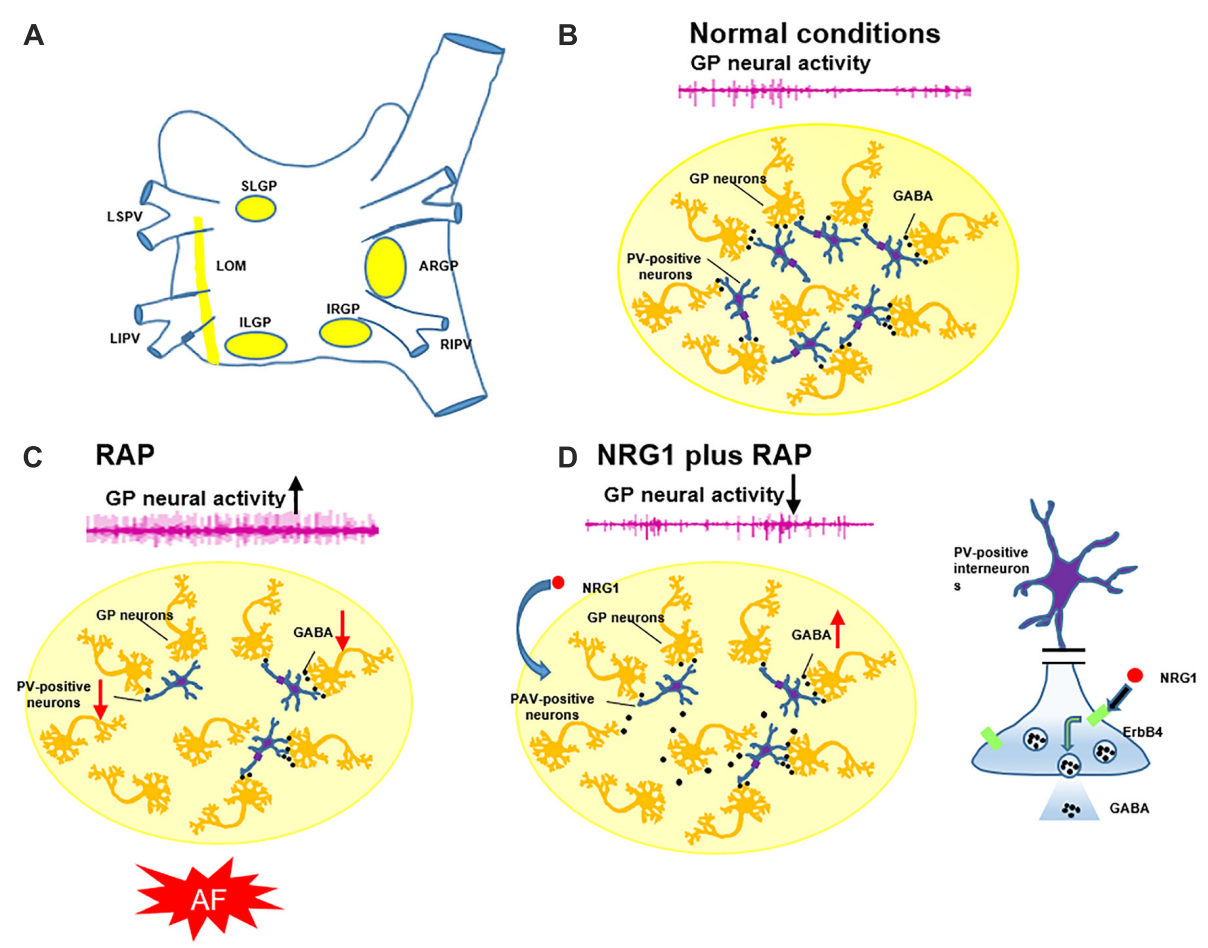

FIGURE 7 | Schematic diagram illustrating the involvement of PAV-positive neurons in the genesis of AF. (A) The position of four major GP and LOM. (B) PAV-positive neurons (inhibitory neurons) play an important role in modulating the GP neural activity in normal conditions. (C) AF simulated by RAP suppressed PAV-positive neurons, diminished evoked GABA release, and increased GP neural activity and promoted AF. (D) Pretreatment with NRG1 activated PAV-positive neurons and enhanced evoked GABA release via ErbB4 receptors, which suppressed RAP-induced GP activation and AF.

risks of serious complications. The present study demonstrated that activation of the NRG1-ErbB4 signaling pathway in the atrial GP was a potential mechanism of GP hyperactivity and a contributing factor to $\mathrm{AF}$ initiation and maintenance. The NRG1-ErbB4 signaling pathway may be a new target for the activation of PAV-positive neurons via the NRG1/ErbB4 pathway and suppression of RAP-induced GP activation, atrial electrical remodeling and AF. Therefore, pharmacological regulation of the NRG1/ErbB4 pathway is a potential treatment for patients in an early stage of AF (e.g., paroxysmal AF).

\section{Study Limitation}

Previous studies demonstrated that the atrial muscle played a role in the NRG1/ErbB4 signaling system (Feron et al., 1999; Zhao et al., 1999). The possibility that the shortening of ERP in response to RAP may be a protective response to limit calcium overload and M2 muscarinic cholinergic receptors activation of nitric oxide synthase in endothelial cells of cardiac muscle may be the physiological mechanism. Isolated atrial myocytes for patch and confocal image evaluations of the impact of NRG1 on myocyte electrophysiology are needed to verify this hypothesis in the future. We did not clearly identify PAV-positive neurons in the ARGP, and whether the other major atrial GP was affected in the same manner was not investigated. Further work must be undertaken to prove the existence of PAV-positive neurons within the cardiac ganglia and its importance and influence on the electrical conduction and propagation of cardiac impulses. Second, the neurochemical profile of the neurons within the intrinsic cardiac nervous system is complex, and we did not block NRG1-ErbB4 signaling by neutralizing NRG1 with ecto-ErbB4 to confirm the effect of NRG1 on GP. Third, we examined the effects of NRG1 on AF in acute canine models, and the long-term effect of NRG1 on AF is not clear. Further studies with long-term follow-up should be performed.

\section{CONCLUSION}

Activation of the NRG1/ErbB4 pathway in atrial GP may be a potential mechanism in suppressing RAP-induced GP activation, atrial electrical remodeling and AF. Pharmacological regulation of the NRG1/ErbB4 pathway is a potential treatment for patients in an early stage of AF.

\section{AUTHOR CONTRIBUTIONS}

$\mathrm{XZ}$, SP, and HJ contributed to the conception of the study. BH, SY, $\mathrm{YW}$, and GM performed the experiments. XZ, ZW, BH, and XS contributed significantly to analysis and manuscript preparation. $\mathrm{XZ}, \mathrm{ZW}$, and $\mathrm{BH}$ performed the data analyses and wrote the manuscript. LY, SP, and HJ helped to perform the analysis with constructive discussions. 


\section{FUNDING}

This work was supported by the National Key R\&D Program of China [2017YFC1307800], the National Natural Science Foundation of China [81470597, 81530011, 81570463, 81600395, and 81700444], the Natural Science Foundation of Hubei

\section{REFERENCES}

Benjamin, E. J., Wolf, P. A., D’agostino, R. B., Silbershatz, H., Kannel, W. B., and Levy, D. (1998). Impact of atrial fibrillation on the risk of death: the Framingham Heart Study. Circulation 98, 946-952. doi: 10.1161/01.CIR.98.10. 946

Buonanno, A., and Fischbach, G. D. (2001). Neuregulin and ErbB receptor signaling pathways in the nervous system. Curr. Opin. Neurobiol. 11, 287-296. doi: 10.1016/S0959-4388(00)00210-5

Chen, P. S., Chen, L. S., Fishbein, M. C., Lin, S. F., and Nattel, S. (2014). Role of the autonomic nervous system in atrial fibrillation: pathophysiology and therapy. Circ. Res. 114, 1500-1515. doi: 10.1161/CIRCRESAHA.114.303772

Corfas, G., Roy, K., and Buxbaum, J. D. (2004). Neuregulin 1-erbB signaling and the molecular/cellular basis of schizophrenia. Nat. Neurosci. 7, 575-580. doi: $10.1038 / \mathrm{nn} 1258$

Dragunow, M., and Robertson, H. A. (1987). Kindling stimulation induces c-fos protein(s) in granule cells of the rat dentate gyrus. Nature 329, 441-442. doi: 10.1038/329441a0

Escher, P., Lacazette, E., Courtet, M., Blindenbacher, A., Landmann, L., Bezakova, G., et al. (2005). Synapses form in skeletal muscles lacking neuregulin receptors. Science 308, 1920-1923. doi: 10.1126/science.1108258

Falls, D. L. (2003). Neuregulins: functions, forms, and signaling strategies. Exp. Cell Res. 284, 14-30. doi: 10.1016/S0014-4827(02)00102-7

Fatini, C., Sticchi, E., Genuardi, M., Sofi, F., Gensini, F., Gori, A. M., et al. (2006). Analysis of $\operatorname{minK}$ and eNOS genes as candidate loci for predisposition to nonvalvular atrial fibrillation. Eur. Heart J. 27, 1712-1718. doi: 10.1093/eurheartj/ ehl087

Fazzari, P., Paternain, A. V., Valiente, M., Pla, R., Lujan, R., Lloyd, K., et al. (2010). Control of cortical GABA circuitry development by Nrg1 and ErbB4 signalling. Nature 464, 1376-1380. doi: 10.1038/nature08928

Feron, O., Smith, T. W., Michel, T., and Kelly, R. A. (1997). Dynamic targeting of the agonist-stimulated $\mathrm{m} 2$ muscarinic acetylcholine receptor to caveolae in cardiac myocytes. J. Biol. Chem. 272, 17744-17748. doi: 10.1074/jbc.272.28. 17744

Feron, O., Zhao, Y. Y., and Kelly, R. A. (1999). The ins and outs of caveolar signaling. $\mathrm{m} 2$ muscarinic cholinergic receptors and eNOS activation versus neuregulin and ErbB4 signaling in cardiac myocytes. Ann. N. Y. Acad. Sci. 874, 11-19. doi: 10.1111/j.1749-6632.1999.tb09220.x

Gonzalez-Burgos, G., Cho, R. Y., and Lewis, D. A. (2015). Alterations in cortical network oscillations and parvalbumin neurons in schizophrenia. Biol. Psychiatry 77, 1031-1040. doi: 10.1016/j.biopsych.2015.03.010

$\mathrm{Hu}, \mathrm{H} ., \mathrm{Gan}$, J., and Jonas, P. (2014). Interneurons. Fast-spiking, parvalbumin(+) GABAergic interneurons: from cellular design to microcircuit function. Science 345:1255263. doi: 10.1126/science. 1255263

Jansen, A. S., Wessendorf, M. W., and Loewy, A. D. (1995). Transneuronal labeling of CNS neuropeptide and monoamine neurons after pseudorabies virus injections into the stellate ganglion. Brain Res. 683, 1-24. doi: 10.1016/00068993(95)00276-V

Katritsis, D. G., Pokushalov, E., Romanov, A., Giazitzoglou, E., Siontis, G. C., Po, S. S., et al. (2013). Autonomic denervation added to pulmonary vein isolation for paroxysmal atrial fibrillation: a randomized clinical trial. J. Am. Coll. Cardiol. 62, 2318-2325. doi: 10.1016/j.jacc.2013.06.053

Kummer, W., and Oberst, P. (1993). Neuronal projections to the guinea pig stellate ganglion investigated by retrograde tracing. J. Auton. Nerv. Syst. 42, 71-80. doi: 10.1016/0165-1838(93)90343-S

Kuramochi, Y., Cote, G. M., Guo, X., Lebrasseur, N. K., Cui, L., Liao, R., et al. (2004). Cardiac endothelial cells regulate reactive oxygen species-induced cardiomyocyte apoptosis through neuregulin-1beta/erbB4 signaling. J. Biol. Chem. 279, 51141-51147. doi: 10.1074/jbc.M408662200
Province [2016CFA065, 2016CFA048, and 2016CFB621], the Health Department Foundation of Zhejiang Province [2012RCB024], the Foundation of Health and Family Planning Commission of Hubei Province [WJ2017C0005], and the Fundamental Research Funds for the Central Universities (Grant Number 2042018kf0102).

Lazarovici, P., Marcinkiewicz, C., and Lelkes, P. I. (2006). Cross talk between the cardiovascular and nervous systems: neurotrophic effects of vascular endothelial growth factor (VEGF) and angiogenic effects of nerve growth factor (NGF)-implications in drug development. Curr. Pharm. Des. 12, 2609-2622. doi: 10.2174/138161206777698738

Lo, L. W., Chang, H. Y., Scherlag, B. J., Lin, Y. J., Chou, Y. H., Lin, W. L., et al. (2016). Temporary suppression of cardiac ganglionated plexi leads to longterm suppression of atrial fibrillation: evidence of early autonomic intervention to break the vicious cycle of "Af Begets Af". J. Am. Heart Assoc. 5:e003309. doi: 10.1161/JAHA.116.003309

Lu, Z., Scherlag, B. J., Lin, J., Niu, G., Fung, K. M., Zhao, L., et al. (2008). Atrial fibrillation begets atrial fibrillation: autonomic mechanism for atrial electrical remodeling induced by short-term rapid atrial pacing. Circ. Arrhythm. Electrophysiol. 1, 184-192. doi: 10.1161/CIRCEP.108.784272

Mei, L., and Nave, K. A. (2014). Neuregulin-ERBB signaling in the nervous system and neuropsychiatric diseases. Neuron 83, 27-49. doi: 10.1016/j.neuron.2014. 06.007

Mei, L., and Xiong, W. C. (2008). Neuregulin 1 in neural development, synaptic plasticity and schizophrenia. Nat. Rev. Neurosci. 9, 437-452. doi: 10.1038/ nrn2392

Meyer, D., and Birchmeier, C. (1995). Multiple essential functions of neuregulin in development. Nature 378, 386-390. doi: 10.1038/378386a0

Park, H. W., Shen, M. J., Lin, S. F., Fishbein, M. C., Chen, L. S., and Chen, P. S. (2012). Neural mechanisms of atrial fibrillation. Curr. Opin. Cardiol. 27, 24-28. doi: 10.1097/HCO.0b013e32834dc4e8

Sha, Y., Scherlag, B. J., Yu, L., Sheng, X., Jackman, W. M., Lazzara, R., et al. (2011). Low-level right vagal stimulation: anticholinergic and antiadrenergic effects. J. Cardiovasc. Electrophysiol. 22, 1147-1153. doi: 10.1111/j.1540-8167. 2011.02070.x

Shao, Q., Liu, H., Ng, C. Y., Xu, G., Liu, E., Li, G., et al. (2014). Circulating serum levels of growth differentiation factor-15 and neuregulin-1 in patients with paroxysmal non-valvular atrial fibrillation. Int. J. Cardiol. 172, e311-e313. doi: 10.1016/j.ijcard.2013.12.173

Shen, M. J., Choi, E. K., Tan, A. Y., Lin, S. F., Fishbein, M. C., Chen, L. S., et al. (2011). Neural mechanisms of atrial arrhythmias. Nat. Rev. Cardiol. 9, 30-39. doi: 10.1038/nrcardio.2011.139

Sheng, X., Scherlag, B. J., Yu, L., Li, S., Ali, R., Zhang, Y., et al. (2011). Prevention and reversal of atrial fibrillation inducibility and autonomic remodeling by low-level vagosympathetic nerve stimulation. J. Am. Coll. Cardiol. 57, 563-571. doi: 10.1016/j.jacc.2010.09.034

Stavrakis, S., Humphrey, M. B., Scherlag, B. J., Hu, Y., Jackman, W. M., Nakagawa, H., et al. (2015). Low-level transcutaneous electrical vagus nerve stimulation suppresses atrial fibrillation. J. Am. Coll. Cardiol. 65, 867-875. doi: 10.1016/j.jacc.2014.12.026

Suzukawa, J., Omori, K., Okugawa, G., Fujiseki, Y., Heizmann, C. W., and Inagaki, C. (1999). Long-lasting c-fos and NGF mRNA expressions and loss of perikaryal parvalbumin immunoreactivity in the development of epileptogenesis after ethacrynic acid-induced seizure. Brain Res. 834, 89-102. doi: 10.1016/S0006-8993(99)01554-1

Tan, A. Y., Zhou, S., Ogawa, M., Song, J., Chu, M., Li, H., et al. (2008). Neural mechanisms of paroxysmal atrial fibrillation and paroxysmal atrial tachycardia in ambulatory canines. Circulation 118, 916-925. doi: 10.1161/ CIRCULATIONAHA.108.776203

Tan, G. H., Liu, Y. Y., Hu, X. L., Yin, D. M., Mei, L., and Xiong, Z. Q. (2011). Neuregulin 1 represses limbic epileptogenesis through ErbB4 in parvalbuminexpressing interneurons. Nat. Neurosci. 15, 258-266. doi: 10.1038/nn. 3005

Thompson, M., Lauderdale, S., Webster, M. J., Chong, V. Z., Mcclintock, B., Saunders, R., et al. (2007). Widespread expression of ErbB2, ErbB3 and ErbB4 
in non-human primate brain. Brain Res. 1139, 95-109. doi: 10.1016/j.brainres. 2006.11.047

Tsai, W. C., Chan, Y. H., Chinda, K., Chen, Z., Patel, J., Shen, C., et al. (2017). Effects of renal sympathetic denervation on the stellate ganglion and brain stem in dogs. Heart Rhythm 14, 255-262. doi: 10.1016/j.hrthm.2016.10.003

Vullhorst, D., Neddens, J., Karavanova, I., Tricoire, L., Petralia, R. S., Mcbain, C. J., et al. (2009). Selective expression of ErbB4 in interneurons, but not pyramidal cells, of the rodent hippocampus. J. Neurosci. 29, 12255-12264. doi: 10.1523/ JNEUROSCI.2454-09.2009

Wake, E., and Brack, K. (2016). Characterization of the intrinsic cardiac nervous system. Auton. Neurosci. 199, 3-16. doi: 10.1016/j.autneu.2016.08.006

Wang, S., Zhou, X., Huang, B., Wang, Z., Zhou, L., Chen, M., et al. (2016). Spinal cord stimulation suppresses atrial fibrillation by inhibiting autonomic remodeling. Heart Rhythm 13, 274-281. doi: 10.1016/j.hrthm.2015. 08.018

Wen, L., Lu, Y. S., Zhu, X. H., Li, X. M., Woo, R. S., Chen, Y. J., et al. (2010). Neuregulin 1 regulates pyramidal neuron activity via ErbB4 in parvalbuminpositive interneurons. Proc. Natl. Acad. Sci. U.S.A. 107, 1211-1216. doi: 10. 1073/pnas.0910302107

Xu, Z., Ford, G. D., Croslan, D. R., Jiang, J., Gates, A., Allen, R., et al. (2005). Neuroprotection by neuregulin-1 following focal stroke is associated with the attenuation of ischemia-induced pro-inflammatory and stress gene expression. Neurobiol. Dis. 19, 461-470. doi: 10.1016/j.nbd.2005.01.027

Yu, L., Huang, B., He, W., Wang, S., Liao, K., Zhou, X., et al. (2014). Spinal cord stimulation suppresses focal rapid firing-induced atrial fibrillation by inhibiting atrial ganglionated plexus activity. J. Cardiovasc. Pharmacol. 64, 554-559. doi: 10.1097/FJC.0000000000000154

Yu, L., Scherlag, B. J., Li, S., Fan, Y., Dyer, J., Male, S., et al. (2013). Low-level transcutaneous electrical stimulation of the auricular branch of the vagus nerve: a noninvasive approach to treat the initial phase of atrial fibrillation. Heart Rhythm 10, 428-435. doi: 10.1016/j.hrthm.2012.11.019

Zhao, Y. Y., Feron, O., Dessy, C., Han, X., Marchionni, M. A., and Kelly, R. A. (1999). Neuregulin signaling in the heart. Dynamic targeting of erbB4 to caveolar microdomains in cardiac myocytes. Circ. Res. 84, 1380-1387. doi: 10.1161/01.RES.84.12.1380

Zhou, S., Chen, L. S., Miyauchi, Y., Miyauchi, M., Kar, S., Kangavari, S., et al. (2004). Mechanisms of cardiac nerve sprouting after myocardial infarction in dogs. Circ. Res. 95, 76-83. doi: 10.1161/01.RES.0000133678.22968.e3

Conflict of Interest Statement: The authors declare that the research was conducted in the absence of any commercial or financial relationships that could be construed as a potential conflict of interest.

Copyright () 2018 Zhou, Wang, Huang, Yuan, Sheng, Yu, Meng, Wang, Po and Jiang. This is an open-access article distributed under the terms of the Creative Commons Attribution License (CC BY). The use, distribution or reproduction in other forums is permitted, provided the original author(s) and the copyright owner(s) are credited and that the original publication in this journal is cited, in accordance with accepted academic practice. No use, distribution or reproduction is permitted which does not comply with these terms. 